\title{
Five-year quality of life in patients with high-risk localized prostate cancer treated with external beam radiotherapy alone versus external beam radiotherapy with high-dose-rate brachytherapy boost: a prospective multicenter study
}

\author{
Evelyn Martínez, MD"*, Olatz Garin, PhD²,3,4, Yolanda Pardo, PhD 2,3,5, Pablo Fernández, MD6, Benjamin Guix MD, PhD7, \\ Cristina Gutiérrez, MD, PhD!', Ana Boladeras, MD, PhD!, Ferran Ferrer, MD, PhD!, Tania Hernández, MD ${ }^{*}$, \\ Adriana Ayala, MD, Mikel Egiguren, MD6, Gema Fernández, MD8, Victor Muñoz, MDª , Victor Macias, MD, PhDº, \\ Joan Pera, MD, PhD', Àngels Pont, B.Stat ${ }^{2,3}$, Montserrat Ferrer, MD, PhD 2,3,5, Ferran Guedea, MD, PhD! \\ and the Multicentric Spanish Group of Clinically Localized Prostate Cancer \\ The Multicentric Spanish Group of Clincally Localized Prostate Cancer: Montse Ferrer, Àngels Pont, Olatz Garin, Yolanda Pardo, Jordi Alonso, \\ Mónica Ávila, Victor Zamora, Ferran Guedea, Montse Ventura, Cristina Gutiérrez, Ferran Ferrer, Ana Boladeras, José Francisco Suárez, \\ Manel Castells, Xavier Bonet, Pastor S, Carmen Bonet, Gemma Sancho, Javier Ponce de León, Joan Palou, Belén de Paula, Alai Goñi, Pablo Fernández, \\ Ismael Herruzo, Asunción Hervás, Alfredo Ramos, Victor Macias, Josep Solé, Marta Bonet, Alfonso Mariño, Patricia Cabrera, Maria José Ortiz \\ 'Servicio de Oncología Radioterápica, Institut Català d'Oncologia, L'Hospitalet de Llobregat, Barcelona, Spain, 2 Unidad de Investigación \\ en Servicios Sanitarios, IMIM-Hospital del Mar, Barcelona, Spain, ${ }^{3}$ CIBER en Epidemiología y Salud Pública (CIBERESP), Madrid, Spain, \\ ${ }^{4}$ Pompeu Fabra University (UPF), Barcelona, Spain, " Universitat Autónoma de Barcelona, Bellaterra, Spain, ${ }^{6}$ Servicio de Oncología \\ Radioterápica, Instituto Oncológico de Guipuzkoa, San Sebastián, Spain, ${ }^{7}$ Fundación IMOR, Institut Mèdic Onco-Radioterapia, \\ Barcelona, Spain, ${ }^{S}$ Servicio de Oncología Radioterápica, Hospital Universitario Central de Asturias, Oviedo, Spain, 'Servicio de Oncología \\ Radioterápica, Hospital do Meixoeiro, Vigo, Spain, ${ }^{10}$ Servicio de Oncología Radioterápica, Hospital Universitari i Politècnic La Fe, Valencia. \\ Spain, and Hospital Universitari General de Catalunya Quiron Salut, Sant Cugat del Vallés, Barcelona, Spain \\ *Present address: Departamento de Radio Oncología, Unidad Médica de Alta Especialidad (UMAE), Centro Médico Nacional de Occidente \\ (CMNO) del Instituto Mexicano del Seguro Social (IMSS), Mexico DF
}

\begin{abstract}
Purpose: Brachytherapy (BT) and external beam radiation therapy (EBRT) are effective treatments for high-risk prostate cancer (PCa). However, the impact of these treatments on health-related quality of life (HRQL) remains unclear. In this study, we compared EBRT alone with EBRT plus a boost with high-dose rate (HDR)-BT to determine the impact on HRQL in patients with high-risk PCa.

Material and methods: Prospective, multicenter study comparing patients with high-risk PCa treated with EBRT alone or EBRT + HDR-BT from 2004 to 2006. HRQL was assessed at baseline (pre-treatment) and periodically over the 5-year follow-up, using the SF-36 (v.2), EPIC, and FACT-G and FACT-P questionnaires.

Results: A total of 129 patients were included in the study, of these, 41 received EBRT alone and 88 EBRT + HDR-BT. All patients received hormonotherapy. Baseline clinical characteristics were similar, except for a slightly higher mean number of comorbidities in the EBRT group. During follow-up, the only significant between-group difference was a greater worsening on EPIC hormonal domain in the EBRT alone group $(p=0.028)$. There were no significant differences in time and interaction of treatment in SF-36, and FACT-G and FACT-P questionnaires or EPIC urinary incontinence, urinary irritative-obstructive, and bowel and sexual domains over the 5-year follow-up. Oncological outcomes were similar in both groups.

Conclusions: After five years of follow-up, EBRT alone or combined with HDR-BT boost had a similar impact on HRQL in patients with high-risk localized PCa. However, patients in the EBRT alone group experienced greater worsening of hormonal domain according to EPIC questionnaire.

Key words: quality of life, prostate cancer, external beam radiotherapy, high-dose-rate brachytherapy.

Address for correspondence: Evelyn Martínez, MD, Servicio de Oncología Radioterápica, Institut Català d'Oncologia (ICO), Gran Via de l'Hospitalet 199-203, E-08908 L'Hospitalet de Llobregat, Barcelona, Spain, phone: +34 932607722, fax: +34 932607725, 凶 e-mail: emperez@iconcologia.net
Received: 27.09 .2020

Accepted: 24.12 .2020

Published: 18.02.2021 


\section{Purpose}

Radical radiotherapy is a well-established treatment option for clinically localized and locally advanced prostate cancer (PCa) [1]. Technological advances in radiotherapy have been rapidly incorporated into clinical practice to reduce treatment-related morbidity and improve oncological outcomes [2,3]. Several randomized trials have demonstrated that dose-escalated radiotherapy-external beam radiotherapy (EBRT) or EBRT plus high-dose-rate (HDR) brachytherapy (BT) boost, improve both local and biochemical control in intermediate- and high-risk PCa $[3,4,5,6]$, despite an increased risk of late genitourinary and gastrointestinal toxicities. The addition of HDR-BT boost allows for highly conformal dose escalation and greater sparing of the surrounding healthy organs [5].

All of the main treatments for PCa have side effects, and radical prostatectomy (RP) seems to produce greater urinary incontinence. BT is associated with an increase in urinary irritative/obstructive symptoms, while EBRT has a greater impact on bowel-related indications $[7,8,9]$. In selected patients, BT is an alternative to RP, limiting the risk of urinary incontinence as well as the potential impact of sexual dysfunction on health-related quality of life (HRQL) [7].

In this context, the main objective of this study was to determine whether EBRT alone or EBRT + HDR-BT was associated with better HRQL outcomes in patients with high-risk PCa, five years after the treatment. Secondary outcome measures included biochemical relapse-free survival (BRFS), overall survival (OS), and cancer-specific survival (CSS) at 5 years.

\section{Material and methods}

\section{Design and study population}

This was a prospective, multicenter study of patients with high-risk PCa, treated at participating centers from 2004 to 2006, and followed for $\geq 5$ years after the treatment. The study protocol was approved by clinical research ethics committees of the six participating hospitals. Written informed consent was obtained from all patients.

Staging and risk group classification were performed using a TNM staging system of the American Joint Committee on Cancer [10] and a risk group classification system developed by D'Amico et al. [11].

Inclusion criteria were biopsy-proven high-risk PCa ( $\geq$ stage T2c, prostate specific antigen [PSA] $>20 \mathrm{ng} / \mathrm{ml}$, or Gleason $>7$ ) without previous transurethral resection.

\section{Clinical evaluation}

Serum PSA levels were measured at all follow-up visits, performed every six months for the first two years and annually thereafter. Biochemical failure was defined as an increase in PSA levels $\geq 2 \mathrm{ng} / \mathrm{ml}$ above the nadir after radiotherapy, in accordance with updated recommendations from the Radiation Therapy Oncology GroupASTRO Phoenix consensus panel [12].

\section{Treatment}

Treatment decisions were made jointly by patients and physicians. All patients received neoadjuvant androgen deprivation therapy (ADT). Adjuvant ADT was prescribed for 2-3 years, in accordance with clinical guidelines [13]. In most cases, patients received an antiandrogen combined with LHRH analogues.

In all cases, EBRT was performed with the patient in supine position, with legs and feet immobilized. All patients underwent a computed tomography (CT) scan in the treatment position. The results of this scan were entered into three-dimensional (3D) treatment planning system and used to contour the prostate, vesicles, bladder, and rectum. External beam clinical target volume (CTV) was defined on CT imaging to cover the prostate gland and seminal vesicles with a $1 \mathrm{~cm}$ margin, except posteriorly, where the margin was reduced to $5 \mathrm{~mm}$ to create planning target volume (PTV). Custom blocking with multileaf collimators was designed using a beam'seye-view, and additional margins were adjusted to provide minimum dose of $95 \%$ to the prostate PTV. Risk organ constraints included the femoral heads (mean dose $\leq 45 \mathrm{~Gy})$ and bladder/rectum $\left(\mathrm{V}_{70}<25 \%\right.$; $\mathrm{V}_{60}$ preferably $<40 \%$, maximum $60 \% ; \mathrm{V}_{40}<60 \%$, maximum $80 \%$ ) [14]. Off-line setup control was assessed weekly by comparing orthogonal portal images with the corresponding digitally-reconstructed radiographs.

External beam radiation therapy was performed using 3D conformal technique by photons from 15 to $18 \mathrm{MV}$ isocentric conformal fields. In most patients, a six-field technique without pelvic irradiation was used. The EBRT alone group received daily fractions of $1.8 / 2 \mathrm{~Gy}, 5$ days per week, for a mean total dose of 73 Gy to the PTV.

For the HDR-BT boost, the patients were placed in lithotomy position under spinal anesthesia. A needle guidance template was attached to an ultrasound probe close to the perineum, and the needles were inserted under transrectal ultrasound guidance. Needle depth was determined by direct visualization on ultrasound and fluoroscopy. A CT scan was performed for volume delineation of the prostate and risk organs. The dose constraints for the HDR-BT boost after EBRT were as follows: PTV with $\mathrm{V}_{100}$ $\geq 98 \%, V_{150} \leq 50 \%$, and $105 \%<D_{90}<115 \%$. The constraints for the rectum were: $D_{2 c c} \leq 75 \%$ and $D_{\max }<100 \%$; and for the urethra: $\mathrm{D}_{2 \%}<120 \%$. The details of radiotherapy technique performed at our center have been published elsewhere [15]. The HDR-BT boost was administered using a temporary iridium-192 implant in one or two fractions separated by 6 hours: 21 patients $(23.5 \%)$ received one fraction (20/2: 9 Gy and 1/21: 9.5 Gy) and 68 patients (76.4\%) received two fractions (dose range, 6-11.5 Gy). For the patients treated with EBRT + HDR-BT, the mean EBRT dose was $51.08 \mathrm{~Gy}$ and the mean BT dose was $17 \mathrm{~Gy}$.

\section{Assessment of HRQL}

Health-related quality of life questionnaires were administered telephonically by trained interviewers with wide expertise on this population, before treatment and during follow-up at 1, 3, 6, 12, 24, 36, 48, and 60 months after the treatment. 
HRQL was evaluated using validated Spanish-language versions of the following instruments: Short form-36 (SF-36), version 2 [16], Functional Assessment of Cancer Therapy - general and prostate (FACT-G and FACT-P) [17], Expanded Prostate Cancer Index composite (EPIC) [18], and International Prostate Symptom Score (IPSS) [19]. The SF-36 contains 36 items with two summary scores: the physical and mental component summary (PCS and MCS), with scores ranging from 0 to 100 on each dimension. The FACT-G (version 4.0) contains 27 items in four dimensions, measuring physical, social, emotional, and functional well-being. The prostate module (FACT-P) is specific for PCa patients and includes 12 questions about urinary symptoms, bowel and sexual function, and pain. Scores range from 0 to 108 on the FACT-G and from $0-48$ on the FACT-P. The 50-item EPIC instrument evaluates four domains (urinary, bowel, sexual, and hormonal), with two urinary scales that distinguish between irritative/obstructive symptoms and incontinence. The final score ranges from 0 to 100 . Higher scores indicate better quality of life (QoL) in all these questionnaires.

The IPSS assesses urinary symptoms, with one question about HRQL. The total score range from 0 to 35, with higher score indicating worse symptoms.

\section{Sample size}

Sample size calculations were based on expected between-group differences on change of HRQL scores as being the principal objective of the present study. It was calculated that a total of 129 patients would be required to detect a difference in change between treatments' groups of 0.5 standard deviation in any HRQL score, given a statistical power of at least $80 \%$ at a significance level of $5 \%$.

\section{Statistical analysis}

Categorical variables were expressed as frequencies and percentages, and continuous variables as mean and standard deviation (SD). Differences in the distribution of variables between the study groups were compared using chisquare $\left(x^{2}\right)$ test or one-way analysis of variance (ANOVA) with post-hoc Tukey's procedure, whenever appropriate.

To assess HRQL changes over time, while accounting for correlation among repeated measures, separate generalized estimating equation (GEE) models were constructed for each specific HRQL score (FACT-P and EPIC) and for the generic ones (SF-36 and FACT-G), all included as dependent variables. Time was included in the model as a categorical variable, and interactions between treatment and time were considered to test differences in trends among treatment groups, after adjusting for age, risk group, and pre-treatment prostate volume.

Differences in BRFS, OS, and CSS at 5 years were analyzed with Kaplan-Meier method and log-rank test. Statistical significance was set at $p<0.05$. SPSS, v.22.0 and SAS/STAT ${ }^{\circledR}$, v.9.4. were used to perform data analyses.

\section{Results}

The study population was comprised of 129 patients, 41 treated with EBTR alone and 88 with EBRT + HDR-BT.
All patients received neoadjuvant ADT. Adjuvant ADT was prescribed for 2-3 years in accordance with clinical guidelines [13], although the final date of ADT administration was not registered.

Table 1 shows patients' clinical characteristics at baseline, mean pre-treatment HRQL scores, and response rate during follow-up. The only statistically significant differences between treatment groups at baseline were a slightly higher mean number of comorbidities in the EBRT group (3.1 with EBRT vs. 2.5 with EBRT + HDR-BT, $p=0.043$ ) and a higher SF-36 PCS score in the EBRT + HDR-BT group (50.8 vs. $53, p=0.04)$. Overall, the response rate to the HRQL questionnaires during the study period was high in both study groups: at 5-years of follow-up, the response rate was 100\% (EBRT) and 97.1\% (EBRT-BT), without significant between-group differences $(p=0.354)$.

Mean changes in QoL scores from baseline to 5-year follow-up are shown in Table 2. Compared with patients treated with EBRT + HDR-BT, the EBRT alone group had significantly lower (worse) hormonal scores (-10.6 vs. -2.4 , respectively, $p=0.028$ ).

Tables 3 and 4 present the results from GEE models constructed for the specific and generic HRQL scores, respectively. For the EBRT alone group, statistically significant differences from baseline were only found 5 years after the treatment for FACT-P $(\beta=-2.4)$, EPIC bowel and hormonal ( $\beta=-3.1$ and $\beta=-10.4$, respectively), among the specific HRQL scores evaluated. No statistically significant differences were found between the two treatment groups, with the exception of a lower impact on the hormonal domain at the EBRT + HDR-BT group.

At 5-years post-treatment, the EBRT alone group showed greater deterioration in SF-36 PCS $(\beta=-9.8$ at years) and in SF-36 MCS ( $\beta=-7.1)$ compared to the combined group. The same pattern was demonstrated by almost all FACT-G domains (Table 3).

Figures 1 and 2 show the differences between groups in the mean HRQL scores during 5-year follow-up. We did not observe any substantial short- or long-term differences between the groups. Moreover, no statistically significant differences in BRFS, OS, and CSS between the study groups were observed (Figures 3-5).

\section{Discussion}

Health-related quality of life is an important outcome measure in patients with prostate cancer. However, longterm differences in HRQL outcomes in these patients have rarely been reported. Most of the available data on HRQL comes from studies, which have assessed the impact of treatment (BT, RP, and EBRT) on low- and intermediate-risk patients $[20,21,22]$. Few studies have compared EBRT alone to EBRT + HDR-BT in high-risk PCa patients in terms of HRQL $[23,24]$.

In our study, we did not observe any significant between-group differences in mean changes in the HRQL questionnaire scores from baseline to 5-year follow-up. However, we did notice a significant difference in the EPIC hormonal domains. Although both groups experienced substantial deterioration in this domain, the EBRT 
Table 1. Patients' characteristics and quality of life scores at pre-treatment evaluation and response rate at each follow-up assessment

\begin{tabular}{|c|c|c|c|}
\hline Variables & $\begin{array}{c}\text { EBRT } \\
(n=41)\end{array}$ & $\begin{array}{c}\text { EBRT + HDR brachytherapy } \\
(n=88)\end{array}$ & $P$-value \\
\hline Age (years), mean (SD) & $71.1(5.6)$ & $70.5(38.5)$ & 0.921 \\
\hline PSA (ng/ml), mean (SD) & $28.7(46.8)$ & $19.8(13.8)$ & 0.103 \\
\hline$\leq 10$ & $12(29.3)$ & $30(34.1)$ & 0.771 \\
\hline $10-20$ & $12(29.3)$ & $21(23.9)$ & \\
\hline$>20$ & $17(41.5)$ & $37(42.0)$ & \\
\hline Gleason score, mean (SD) & $7.3(0.8)$ & $7.0(1.1)$ & 0.142 \\
\hline$<7$ & $6(15.0)$ & $27(30.7)$ & 0.060 \\
\hline$\geq 7$ & $34(85.0)$ & $61(69.3)$ & \\
\hline \multicolumn{4}{|l|}{ Clinical T stage, $n(\%)$} \\
\hline $\mathrm{T} 1$ & $5(12.2)$ & $6(6.8)$ & 0.581 \\
\hline $\mathrm{T} 2$ & $15(36.6)$ & $36(40.9)$ & \\
\hline T3 & $21(51.2)$ & $46(52.3)$ & \\
\hline Prostate volume, mean (SD) & $44.6(29.8)$ & $40.5(19.0)$ & 0.520 \\
\hline Comorbidities, $n(\%)$ & $3.1(1.7)$ & $2.5(1.5)$ & 0.043 \\
\hline 0 & $1(2.4)$ & $8(9.1)$ & 0.465 \\
\hline 1 & $7(17.1)$ & $19(21.6)$ & \\
\hline 2 & $8(19.5)$ & $14(15.9)$ & \\
\hline 3 & $25(61.0)$ & $47(53.4)$ & \\
\hline \multicolumn{4}{|l|}{ Quality of life scores, mean (SD) } \\
\hline SF-36 PCS & $50.8(7.2)$ & $53.0(4.4)$ & 0.040 \\
\hline SF-36 MCS & $56.2(5.3)$ & $55.3(5.3)$ & 0.362 \\
\hline FACT-G & $79.2(10.0)$ & $79.6(7.4)$ & 0.777 \\
\hline FACT-P & $38.0(5.2)$ & $39.0(4.0)$ & 0.237 \\
\hline FACT physical well-being & $26.4(2.4)$ & $26.7(1.8)$ & 0.453 \\
\hline FACT social well-being & $17.5(3.9)$ & $17.8(2.9)$ & 0.613 \\
\hline FACT emotional well-being & $19.2(3.7)$ & $18.9(3.3)$ & 0.677 \\
\hline FACT functional well-being & $16.2(3.8)$ & $16.3(3.3)$ & 0.954 \\
\hline \multicolumn{4}{|l|}{ EPIC urinary } \\
\hline Incontinence & $90.8(17.2)$ & $94.8(14.6)$ & 0.181 \\
\hline Irritative/obstructive & $94.1(9.1)$ & $93.2(10.3)$ & 0.662 \\
\hline EPIC bowel & $97.8(7.6)$ & $98.5(3.6)$ & 0.477 \\
\hline EPIC sexual & $38.6(23.2)$ & $37.2(23.4)$ & 0.742 \\
\hline EPIC hormonal & $93.7(8.6)$ & $90.8(10.6)$ & 0.135 \\
\hline IPSS & $9.0(7.9)$ & $6.6(6.5)$ & 0.078 \\
\hline \multicolumn{4}{|c|}{ Response rate HRQL questionnaires, $n(\%)$} \\
\hline Pre-treatment & 41/41 (100.0) & $88 / 88(100.0)$ & \\
\hline Follow-up, month 1 & $34 / 41(82.9)$ & $50 / 88(56.8)$ & 0.004 \\
\hline Follow-up, month 3 & 40/41 (97.6) & 78/88 (88.6) & 0.091 \\
\hline Follow-up, month 6 & $39 / 40(97.5)$ & $81 / 87(93.1)$ & 0.313 \\
\hline Follow-up, month 12 & $39 / 40(97.5)$ & 79/86 (91.9) & 0.227 \\
\hline Follow-up, month 24 & 39/39 (100.0) & $79 / 83(95.2)$ & 0.163 \\
\hline Follow-up, month 36 & $34 / 37(91.9)$ & $65 / 82(79.3)$ & 0.088 \\
\hline Follow-up, month 48 & $24 / 33(72.7)$ & 73/77 (94.8) & 0.001 \\
\hline Follow-up, month 60 & 29/29 (100.0) & $67 / 69(97.1)$ & 0.354 \\
\hline
\end{tabular}

EBRT - external beam radiotherapy, HDR - high-dose-rate, SF-36- medical outcomes study 36-item short form, PCS - physical component summary, MCS - mental component summary, FACT-G and FACT-P - Functional Assessment of Cancer Therapy (general and prostate module), EPIC - Expanded Prostate Cancer Index composite, IPSS - International Prostate Symptom Score. Data as frequencies and percentages in parenthesis unless otherwise stated 
Table 2. Mean differences in scores in HRQL questionnaires at 5 years of follow-up as compared with baseline

\begin{tabular}{lccc} 
Variables & $\begin{array}{c}\text { EBRT } \\
(n=41)\end{array}$ & $\begin{array}{c}\text { EBRT + HDR brachytherapy } \\
(n=88)\end{array}$ & $P$-value \\
\hline SF-36 PCS & $-10.2(8.5)$ & $-7.2(8.7)$ & 0.121 \\
\hline SF-36 MCS & $-6.3(11.1)$ & $-2.7(10.5)$ & 0.131 \\
\hline FACT physical well-being & $-3.0(4.9)$ & $-1.9(3.7)$ & 0.212 \\
\hline FACT social well-being & $-2.1(3.6)$ & $-1.6(2.8)$ & 0.433 \\
\hline FACT emotional well-being & $-2.5(4.0)$ & $-1.6(3.6)$ & 0.290 \\
\hline FACT functional well-being & $-1.0(5.2)$ & $1.0(4.6)$ & 0.062 \\
\hline FACT-G & $-8.7(12.5)$ & $-4.2(10.2)$ & 0.064 \\
\hline FACT-P & $-2.6(6.3)$ & $-2.6(5.7)$ & 0.989 \\
\hline EPIC domains & & & 0.238 \\
\hline Urinary incontinence & $-6.1(24.7)$ & $-12.8(24.8)$ & 0.416 \\
\hline Urinary irritative/obstructive & $-4.8(22.7)$ & $-8.6(19.1)$ & 0.614 \\
\hline Bowel & $-2.5(7.2)$ & $-3.6(11.1)$ & 0.798 \\
\hline Sexual & $0.8(30.1)$ & $-0.8(26.4)$ & 0.028 \\
\hline Hormonal & $-10.6(17.2)$ & $-2.4(16.2)$ & 0.263
\end{tabular}

EBRT - external beam radiotherapy, HDR - high-dose-rate, SF-36-medical outcomes study 36-item short form, PCS - physical component summary, MCS - mental component summary, FACT-G and FACT-P - Functional Assessment of Cancer Therapy (general and prostate module), EPIC - Expanded Prostate Cancer Index composite, IPSS - International Prostate Symptom Score. Data expressed as mean difference (standard deviation, SD)

alone group had significantly lower (worse) hormonal scores at 5-year follow-up. Moreover, the GEE model revealed greater worsening mainly in the FACT-P, FACT-G, SF-36, and bowel domain in the EBRT group in the fifthyear post-treatment. The greater deterioration of hormonal and sexual scores was most marked until the second year after the treatment. After this time point, there was a trend towards an improvement in these scores, probably due to the finalization of ADT and the consequent improvement in ADT-related side effects. This finding could be due to the slightly better $(p=0.135)$ baseline EPIC hormonal summary scores in the EBRT group.

Overall, findings of this study suggest that EBRT alone or combined with HDR-BT appear to have similar effects on HRQL. However, in our sample, patients in the BT boost group had fewer comorbidities at baseline, which could partially explain why we observed no major between-group differences. Indeed, this probably also explains why they were eligible for HDR-BT boost. Further studies with longer series and longer follow-up would provide more information about the impact of these radiotherapy modalities on HRQL.

Dose escalation with EBRT followed by brachytherapy boost have been shown to improve BRFS $[5,15,25]$. In the ASCENDE-RT trial [26], patients were randomized to dose-escalated EBRT (78 Gy) or EBRT plus low-doserate BT boost. The patients in the boost group were twice as likely to be free of biochemical failure at a median of 6.5 years of follow-up, without significant differences in OS. Theoretically, dose escalation with HDR allows for an increase in biologically-effective dose, improving tumor control and sparing organs at risk [22]. However, in our series, we found no statistical significance between-group differences in BRFS, OS, or CSS. Moreover, our BRFS results differ from those reported in randomized trials [22,23,25,26], probably because of the small number of patients. Certainly, the lack of significant differences in survival outcomes in our study may be due to the limited sample size, which was powered to detect differences in the main study variable (HRQL), but not for survival outcomes.

Other authors, such as Ferrer et al. [18], have reported that the addition of ADT causes temporary deterioration in some HRQL domains, a finding that is consistent with the worsening observed in our patients in the EPIC hormonal domain. Those authors observed that ADT was associated with worse results related to vitality, hormonal function, and sexuality. Only a few studies with long follow-up have been performed to assess HRQL in patients with high-risk PCa [22,27]. In this group of patients, it is necessary to achieve the most appropriate treatment to improve local control with the lowest toxicity and the best possible HRQL.

The main limitations of our study are the relatively small study population, the differences in group size, and the lack of randomization. Another limitation is the use of conformal 3D-RT rather than more advanced techniques, such as intensity modulated radiation therapy (IMRT) or volumetric modulated arc therapy (VMAT). However, during the study period (2004-2006), neither of those techniques was available in the participating hospitals. Nevertheless, given the similar clinical characteristics at baseline in the two groups, the comparison can be considered valid. Study strengths include the prospective design and the fact that the same two trained interviewers administered all HRQL questionnaires during the entire period of study.

\section{Conclusions}

In the present study, EBRT administered alone or in combination with HDR-BT boost had a similar impact on 


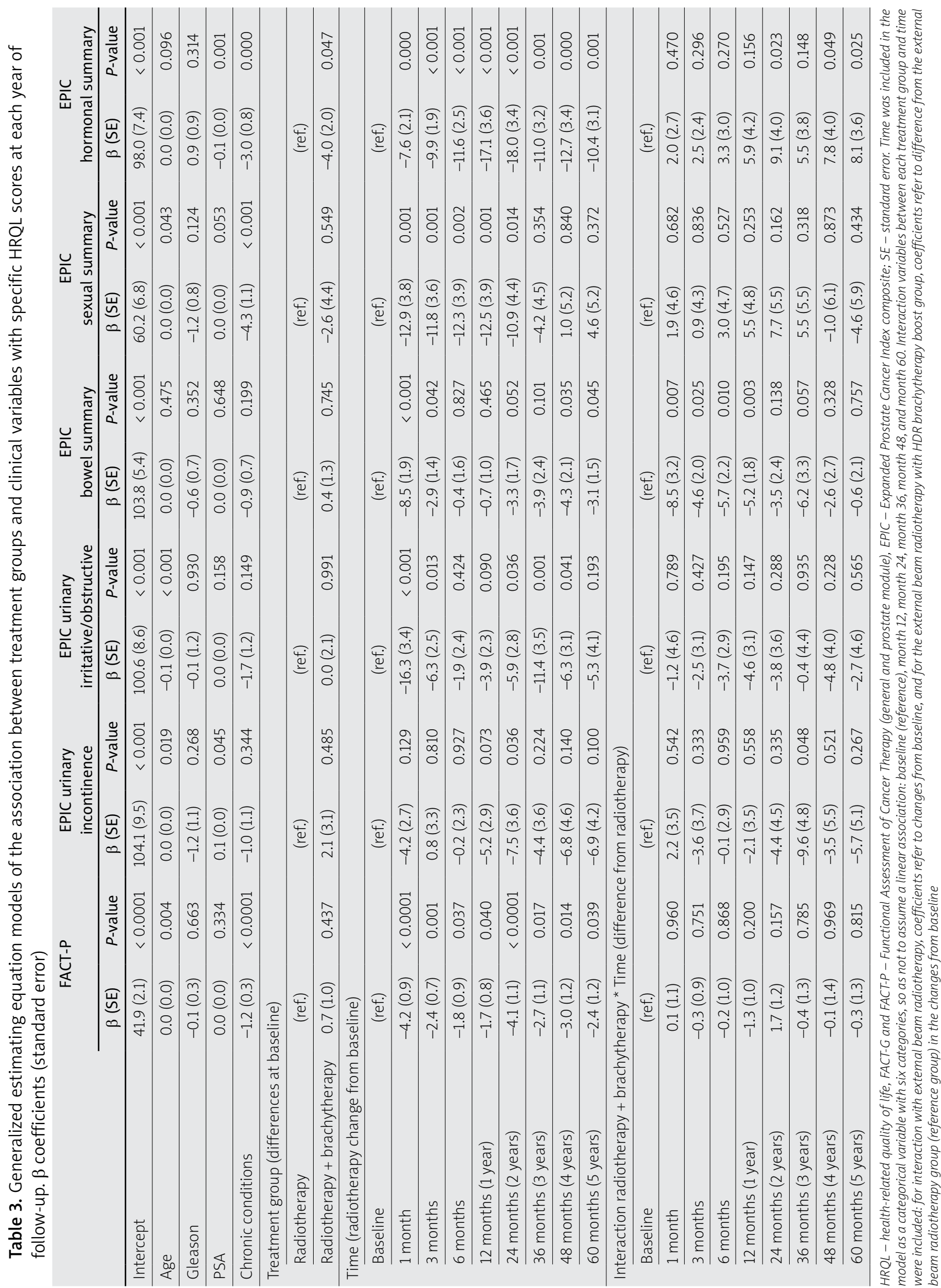




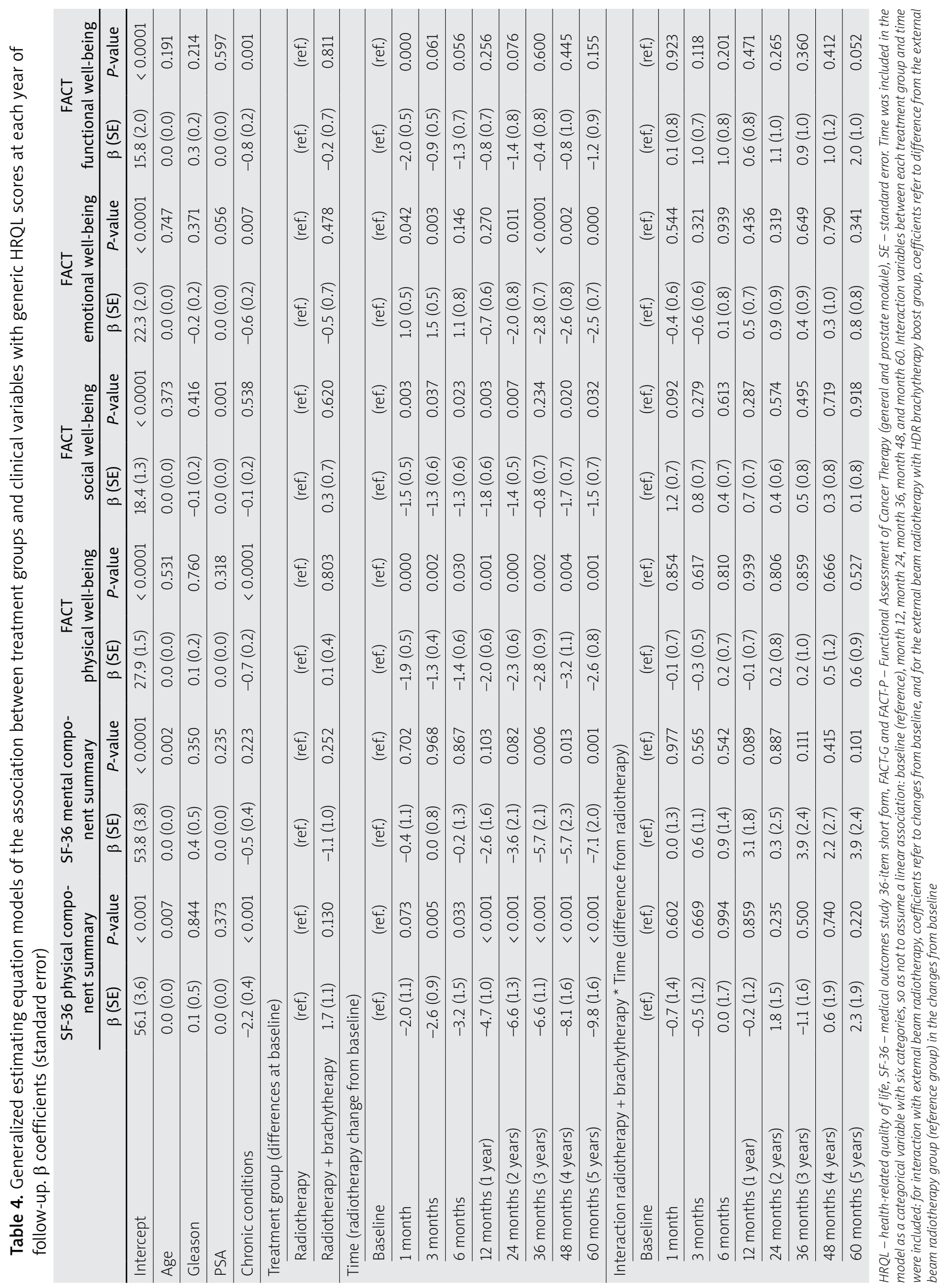


A

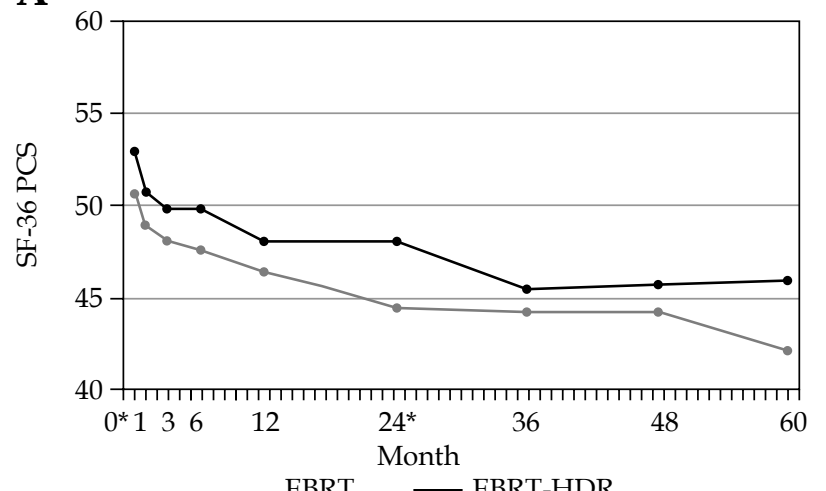

C

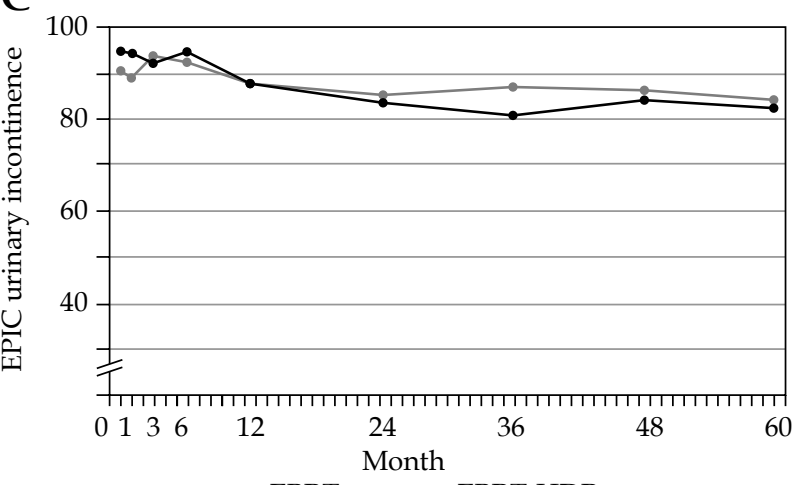

E
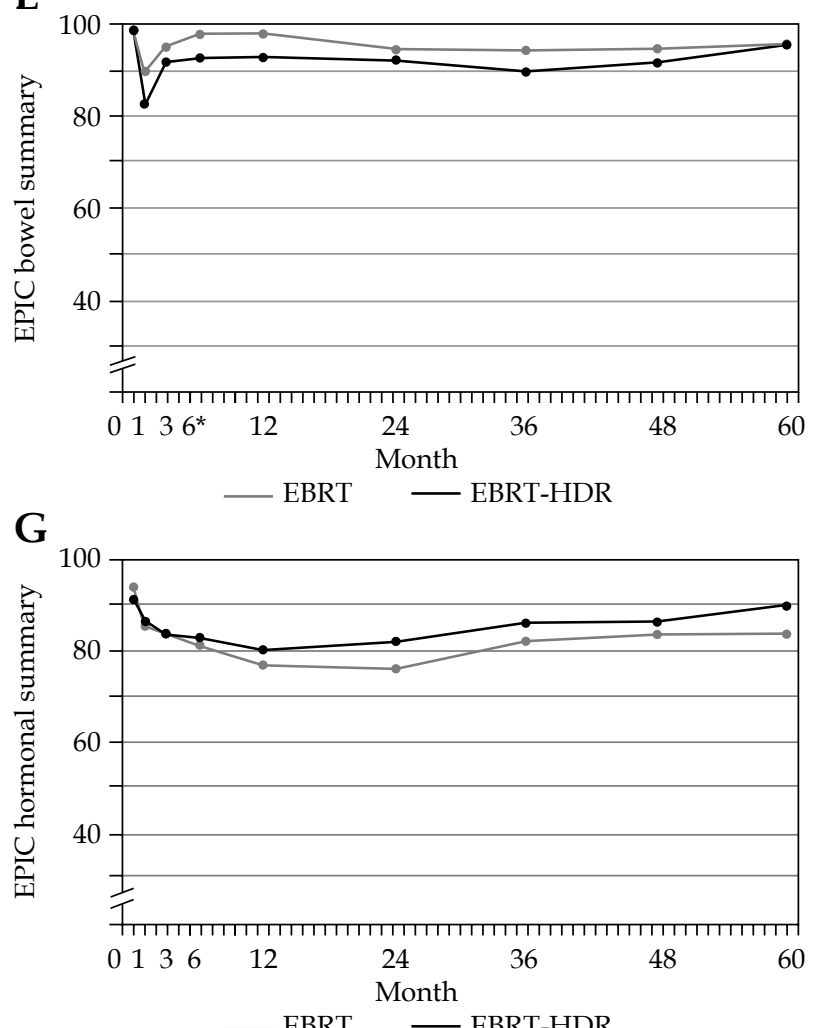

B

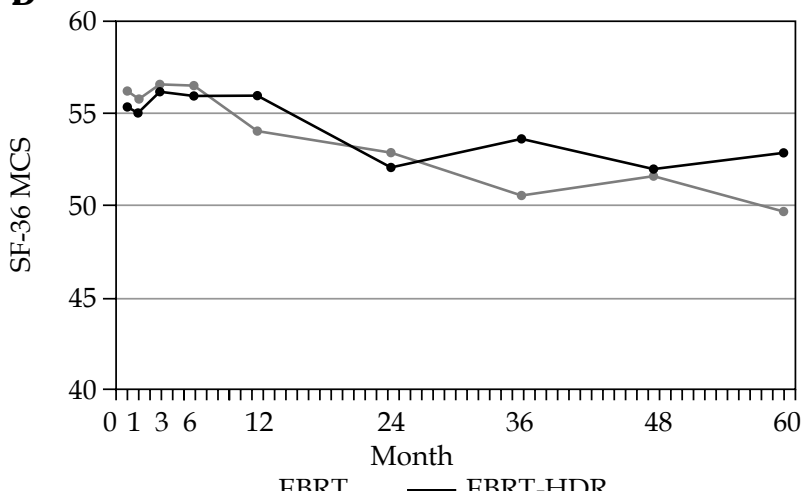

D

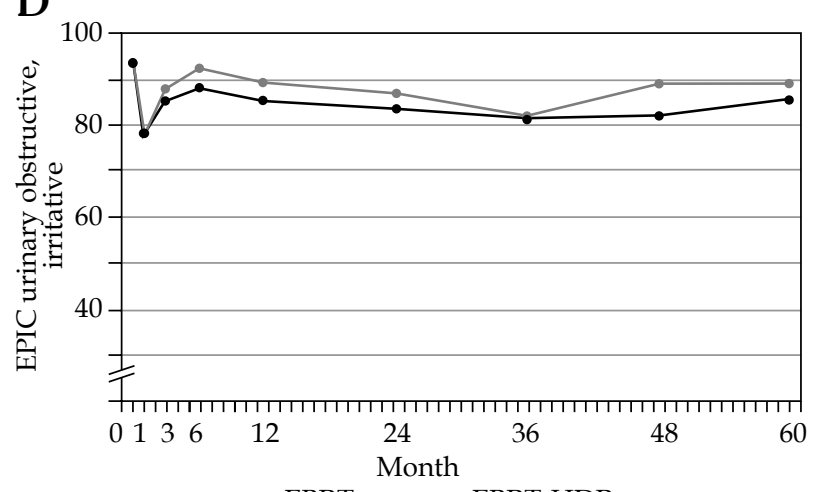

— EBRT — EBRT-HDR

F

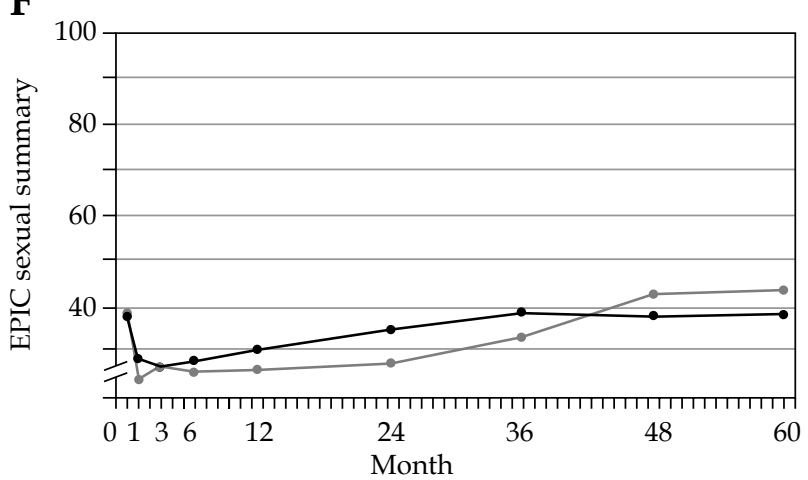

H

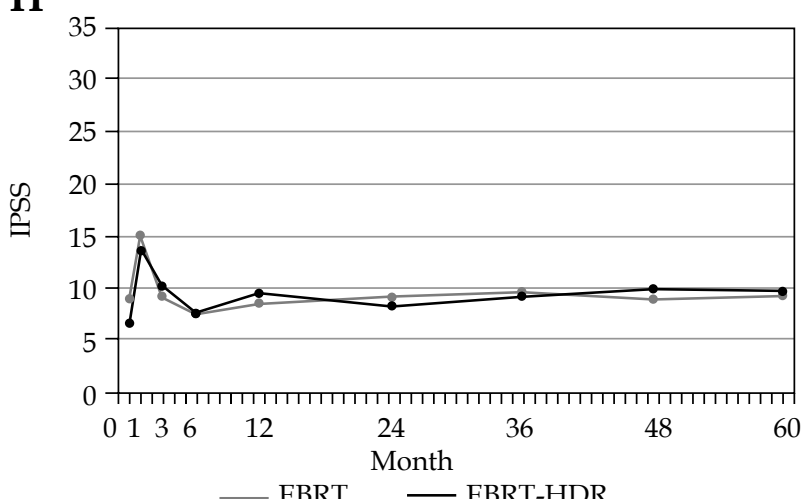

— EBRT - EBRT-HDR

Fig. 1. Differences between EBRT alone and EBRT combined with HDR brachytherapy regarding the impact on mean scores of HRQL questionnaires during the 5 years of follow-up for short form-36 (SF-36) physical component score (PCS; A); SF-36 mental component score (MCS; B); expanded prostate cancer index composite (EPIC) urinary incontinence (C), urinary obstructive/irritative (D), bowel (E), sexual (F), hormonal domains (G), and international prostate symptom score (IPSS; H); one-way analysis of variance of QoL scores among the two treatment groups for each follow-up assessment; Tukey's studentized range pos-hoc comparisons: ${ }^{*} p<0.05$ for external-beam radiotherapy (grey) vs. external-beam radiotherapy combined with HDR brachytherapy boost (black) 
A

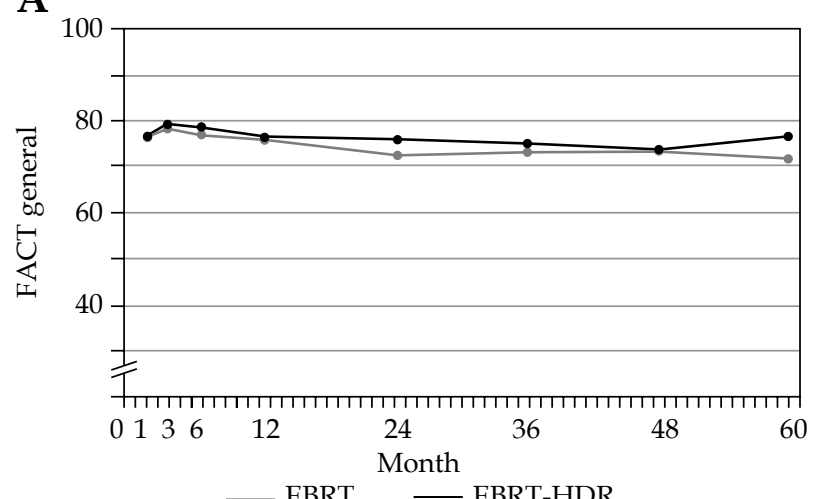

C

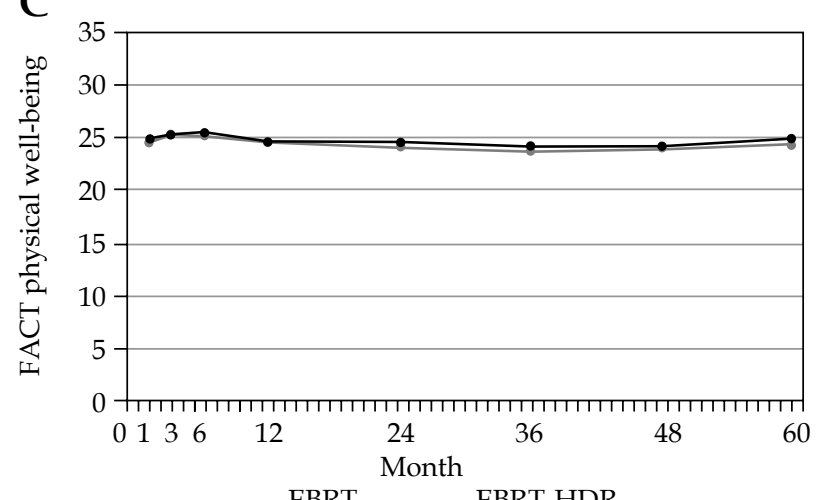

E

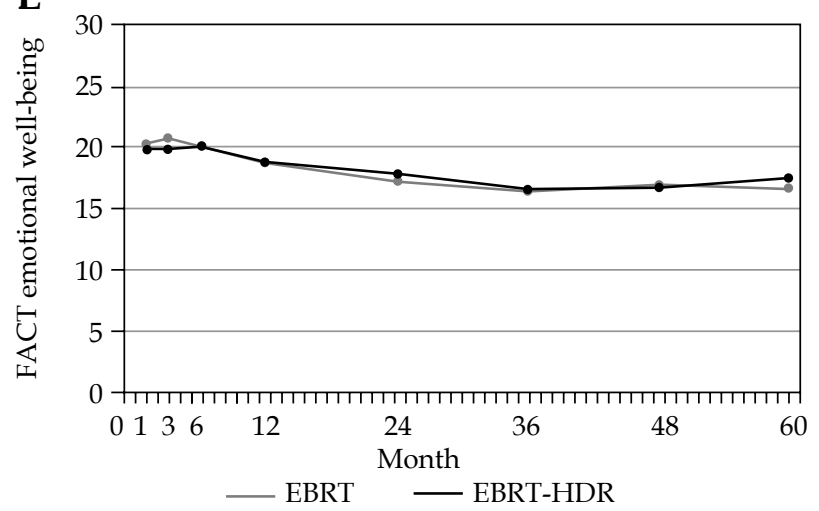

B

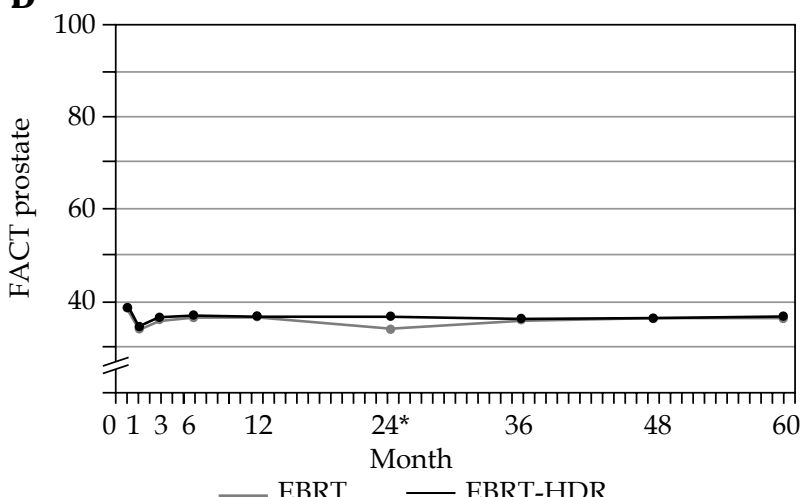

D

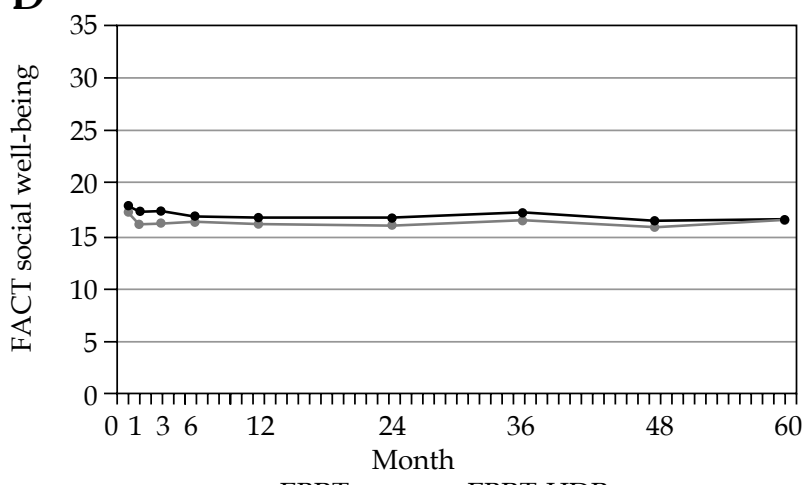

— EBRT — EBRT-HDR

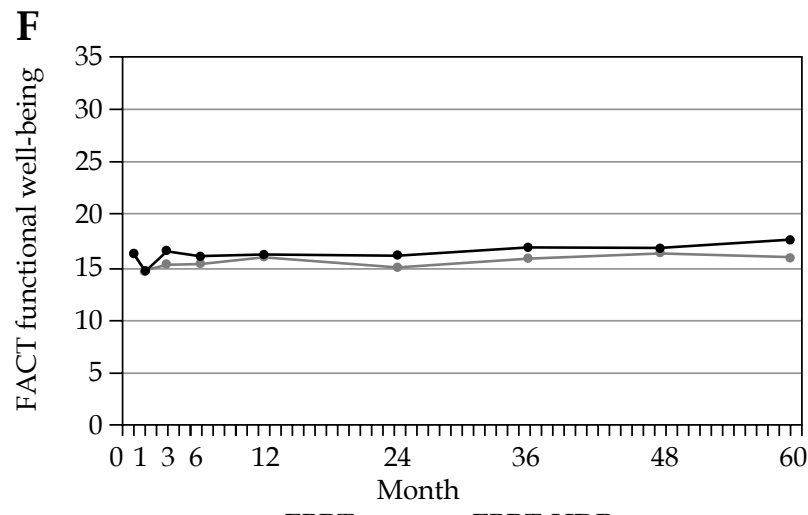

— EBRT — EBRT-HDR

Fig. 2. Differences between EBRT alone and EBRT combined with HDR brachytherapy regarding the impact on mean scores in HRQL questionnaires during 5 years of follow-up for functional assessment cancer therapy-general (FACT-general; A) and prostate-specific (FACT-prostate; B); FACT physical well-being (C); FACT social well-being (D); FACT emotional well-being (E) and FACT functional well-being (F). One-way analysis of variance of QoL scores among the two treatment groups for each follow-up assessment. Tukey's studentized range post-hoc comparisons: ${ }^{*} p<0.05$ for external-beam radiotherapy (grey) vs. external-beam radiotherapy combined with HDR brachytherapy boost (black)

HRQL in patients with high-risk localized PCa over 5-year follow-up. However, patients in the EBRT alone group experienced significantly greater worsening in hormonal symptoms (EPIC questionnaire) at 5-year follow-up, per- haps due to the higher EPIC hormonal summary scores at baseline in this group. Longer follow-up would be needed to minimize the effects of ADT on HRQL and to determine the best treatment in terms of HRQL. 


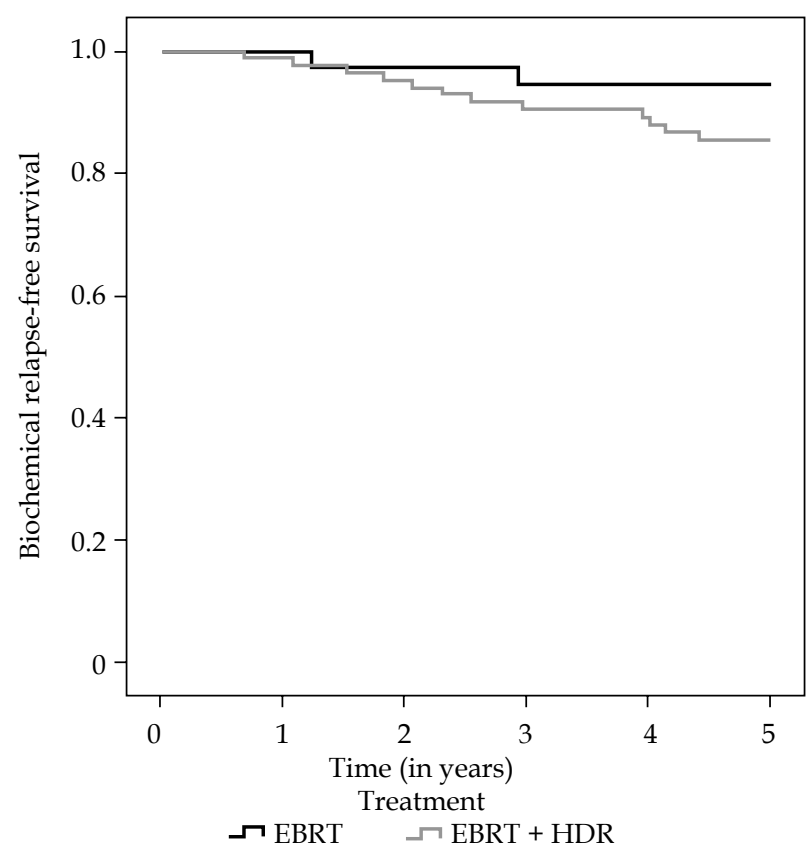

Fig. 3. Biochemical relapse-free survival curves in patients treated with EBRT alone and EBRT + HDR-BT. Kaplan-Meier method and log-rank test: ${ }^{*} p<0.05$ for external-beam radiotherapy (black) vs. external-beam radiotherapy combined with HDR brachytherapy boost (grey)

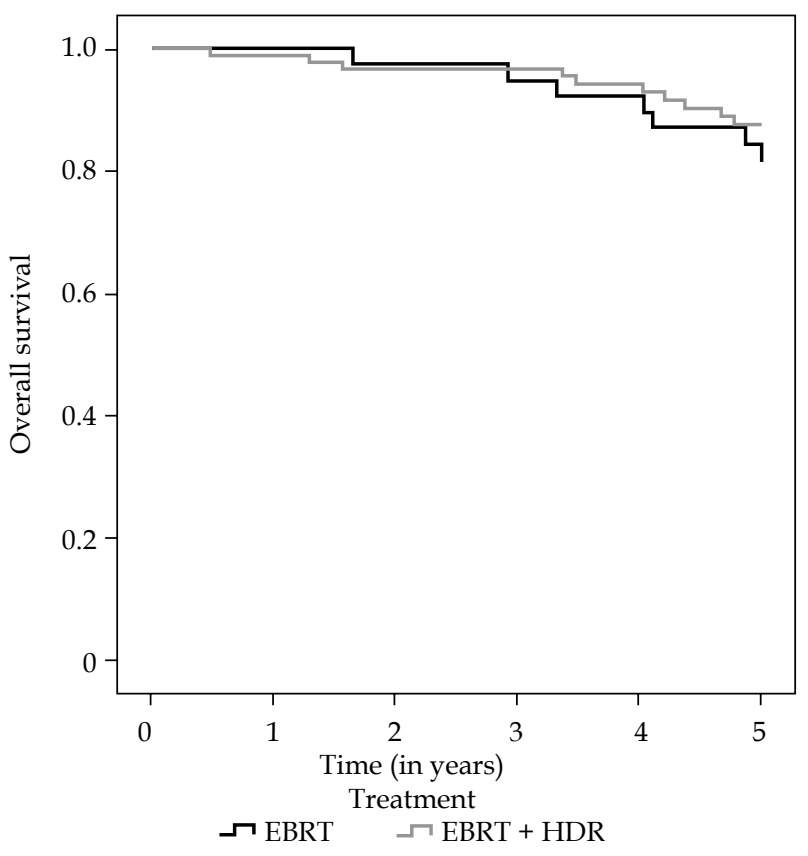

Fig. 4. Overall survival curves in patients treated with EBRT alone and EBRT + HDR-BT. Kaplan-Meier method and log-rank test: ${ }^{*} p<0.05$ for external-beam radiotherapy (black) vs. external-beam radiotherapy combined with HDR brachytherapy boost (grey)

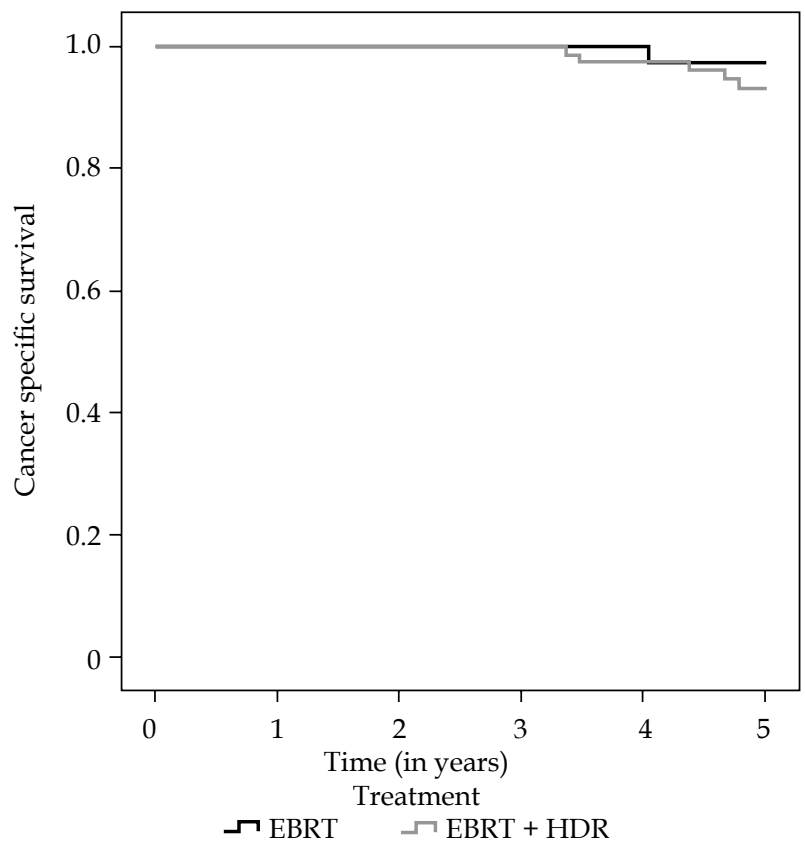

Fig. 5. Cancer-specific survival curves in patients treated with EBRT alone and EBRT + HDR-BT. Kaplan-Meier method and log-rank test: ${ }^{*} p<0.05$ for external-beam radiotherapy (black) vs. external-beam radiotherapy combined with HDR brachytherapy boost (grey)

\section{Acknowledgments}

The authors would like to thank Marta Pulido, MD, and Bradley Londres for professional English language editing.

\section{Disclosure}

The authors report no conflict of interest.

\section{References}

1. Chen RC, Basak R, Meyer AM et al. Association between choice of radical prostatectomy, external beam radiotherapy, brachytherapy, or active surveillance and patient-reported quality of life among men with localized prostate cancer. JAMA 2017; 317: 1141-1150.

2. Sheets NC, Goldin GH, Meyer AM et al. Intensity-modulated radiation therapy, proton therapy, or conformal radiation therapy and morbidity and disease control in localized prostate cancer. JAMA 2012; 307: 1611-1620.

3. Hamdy FC, Donovan JL, Lane JA et al. 10-year outcomes after monitoring, surgery, or radiotherapy for localized prostate cancer. N Engl J Med 2016; 375: 1415-1424.

4. Kee DLC, Gal J, Falk AT et al. Brachytherapy versus external beam radiotherapy boost for prostate cancer: Systematic review with meta-analysis of randomized trials. Cancer Treat Rev 2018; 70: 265-271.

5. Hoskin PJ, Rojas AM, Bownes PJ et al. Randomised trial of external beam radiotherapy alone or combined with highdose-rate brachytherapy boost for localised prostate cancer. Radiother Oncol 2012; 103: 217-222.

6. Sathya JR, Davis IR, Julian JA et al. Randomized trial comparing iridium implant plus external-beam radiation therapy 
with external-beam radiation therapy alone in node-negative locally advanced cancer of the prostate. J Clin Oncol 2005; 23: 1192-1199.

7. Martinez E, Daidone A, Gutierrez C et al. Permanent seed brachytherapy for clinically localized prostate cancer: Longterm outcomes in a 700 patient cohort. Brachytherapy 2015; 14: $166-172$.

8. Ávila M, Becerra V, Guedea F et al. Estimating preferences for treatments in patients with localized prostate cancer. Int J Radiat Oncol Biol Phys 2015; 91: 277-287.

9. Ferrer M, Guedea F, Suárez JF et al. Quality of life impact of treatments for localized prostate cancer: Cohort study with a 5 year follow-up. Radiother Oncol 2013; 108: 306-313.

10. Edge SB, Compton CC. The American joint committee on cancer: The 7th edition of the AJCC cancer staging manual and the future of TNM. Ann Surg Oncol 2010; 17: 1471-1474.

11. D'Amico A, Altschuler M, Whittington $R$ et al. The use of clinical parameters in an interactive statistical package to predict pathological features associated with local failure after radical prostatectomy for prostate cancer. Clin Perform Qual Health Care 1993; 1: 219-222.

12. Roach M, Hanks G, Thames H et al. Defining biochemical failure following radiotherapy with or without hormonal therapy in men with clinically localized prostate cancer: Recommendations of the RTOG-ASTRO Phoenix Consensus Conference. Int J Radiat Oncol Biol Phys 2006; 65: 965-974.

13. Bolla M, Collette L, Blank L et al. Long-term results with immediate androgen suppression and external irradiation in patients with locally advanced prostate cancer (an EORTC study): A phase III randomised trial. Lancet 2002; 360: 103-108.

14. Fiorino C, Cozzarini C, Vavassori V et al. Relationships between DVHs and late rectal bleeding after radiotherapy for prostate cancer: Analysis of a large group of patients pooled from three institutions. Radiother Oncol 2002; 64: 1-12.

15. Boladeras A, Santorsa L, Gutierrez C et al. External beam radiotherapy plus single-fraction high dose rate brachytherapy in the treatment of locally advanced prostate cancer. Radiother Oncol 2014; 112: 227-232.

16. Alonso J, Prieto L, Antó AJ. La versión española del SF-36 Health Survey (Cuestionario de Salud SF-36): un instrumento para la medida de los resultados clínicos. Med Clin 1995; 104: 771-776.

17. Cella D, Hernandez L, Bonomi AE et al. Spanish language translation and initial validation of the functional assessment of cancer therapy quality-of-life instrument. Med Care 1998; 36: $1407-1418$

18. Ferrer M, Garin O, Pera J et al. Evaluation of the quality of life of patients with localizad prostate cancer: validation of the Spanish version of the EPIC. Med Clin (Barc) 2009; 132: 128-135.

19. Badia X, Garcia-Losa M, Dal-Re R. Ten-language translation and harmonization of the international prostate symptom score: Developing a methodology for multinational clinical trials. Eur Urol 1997; 31: 129-140.

20. Ferrer M, Suárez JF, Guedea F et al. Health-related quality of life 2 years after treatment with radical prostatectomy, prostate brachytherapy, or external beam radiotherapy in patients with clinically localized prostate cancer. Int J Radiat Oncol Biol Phys 2008; 72: 421-432.

21. Sanda MG, Dunn RL, Michalski J et al. Quality of life and satisfaction with outcome among prostate-cancer survivors. N Engl J Med 2008; 358: 1250-1261.

22. Hoskin PJ, Rojas AM, Ostler PJ et al. Quality of life after radical radiotherapy for prostate cancer: longitudinal study from a randomised trial of external beam radiotherapy alone or in combination with high dose rate brachytherapy. Clin Oncol 2013; 25: 321-327.
23. Donovan JL, Hamdy FC, Lane JA et al. Patient-reported outcomes after monitoring, surgery, or radiotherapy for prostate cancer. N Engl J Med 2016; 375: 1425-1437.

24. Giberti C, Chiono L, Gallo F et al. Radical retropubic prostatectomy versus brachytherapy for low-risk prostatic cancer: A prospective study. World J Urol 2009; 27: 607-612.

25. Dayes IS, Parpia S, Gilbert J et al. Long-term results of a randomized trial comparing iridium implant plus external beam radiation therapy with external beam radiation therapy alone in node-negative locally advanced cancer of the prostate. Int J Radiat Oncol Biol Phys 2017; 99: 90-93.

26. Morris WJ, Tyldesley S, Rodda S et al. Androgen Suppression Combined with Elective Nodal and Dose Escalated Radiation Therapy (the ASCENDE-RT Trial): an analysis of survival endpoints for a randomized trial comparing a lowdose-rate brachytherapy boost to a dose-escalated external beam boost for high- and intermediate-risk prostate cancer. Int J Radiat Oncol Biol Phys 2017; 98: 275-285.

27. Miller DC, Sanda MG, Dunn RL et al. Long-term outcomes among localized prostate cancer survivors: Health-related quality-of-life changes after radical prostatectomy, external radiation, and brachytherapy. J Clin Oncol 2005; 23: 2772-2780. 